\title{
The Impact of the Autonomous Vehicles' Penetration on the Road Accidents Involving Vulnerable Road Users
}

\author{
János Juhász \\ Budapest University of Technology and Economics \\ Faculty of Transportation Engineering and Vehicle Engineering \\ Müegyetem rakpart 3., Budapest, Hungary \\ Janos.Juhasz@mail.bme.hu
}

The traffic of the autonomous vehicles will fundamentally change the road transport. This transformation will be a slow process, estimated for several decades. One of the biggest challenges is whether the autonomous vehicles and the vulnerable road users can travel together, if so, how? The problem is not just a technological task. The vehicles' development is very fast and it has important results, but it should be noted that this development can also lead to significant changes in traffic patterns and transport behaviour. For example, it is expected that in urban transport the use of car-sharing systems will increase instead of the usage of the own cars.

The purpose of the research is to investigate the evolution of the frequency of the most typical accident types and primary cause (supposed by Police officer) as a consequence of the autonomous vehicles' penetration. Furthermore, it's an additional aim of this research to try to find new accident types or primary accident causes.

The research was based on the official statistical database in Hungary, the road traffic accidents involving personal injury. The categorization of the accident types and primary causes was the same as in the official statistics.

The most typical and critical accident types are presented in form of analysis of real accidents.

Currently, the development of the control of autonomous vehicles is aimed at expanding human perception and action capabilities and the development of strategies is oriented to the imitation of human thinking. This is a very complicated task, in fact the traffic of autonomous vehicles would require a completely different, simpler strategy.

The presentation also intends to point out that expectations of autonomous vehicles are often excessive in relation to road safety.

In conclusion, the presentation sets out questions that need to be answered based on further research. 